\title{
Sensibilisation lexico-grammaticale à l'aide d'un outil de concordance
}

John Osborne

\section{OpenEdition}

\section{Journals}

Édition électronique

URL : http://journals.openedition.org/asp/3695

DOI : 10.4000/asp.3695

ISSN : 2108-6354

\section{Éditeur}

Groupe d'étude et de recherche en anglais de spécialité

\section{Édition imprimée}

Date de publication : 1 décembre 1996

Pagination : 417-422

ISSN : 1246-8185

\section{Référence électronique}

John Osborne, «Sensibilisation lexico-grammaticale à l'aide d'un outil de concordance », ASp [En ligne], 11-14 | 1996, mis en ligne le 24 juillet 2013, consulté le 01 mai 2019. URL : http:// journals.openedition.org/asp/3695 ; DOI : 10.4000/asp.3695

Ce document a été généré automatiquement le 1 mai 2019.

Tous droits réservés 


\title{
Sensibilisation lexico-grammaticale à l'aide d'un outil de concordance
}

\author{
John Osborne
}

\section{Le problème des erreurs fossilisées}

1 Pourquoi certaines erreurs résistent-elles farouchement à la correction? Les erreurs que nous disons ainsi "fossilisées" (Selinker 1972) possèdent deux caractéristiques marquantes : leur longévité et leur capacité à se maintenir jusque dans les styles les plus surveillés. En voici quelques exemples, relevés dans les productions d'apprenants relativement avancés :

\footnotetext{
As the grid illustrates, you cannot expect a high degree of control since you are not responsible for the sales. One advantage is that it does not require an important capital investment.

As for opportunities, they mainly depend on the product. The question is: is cookware interesting for distributors?

German people have the Northern state of mind. Indeed, the German manager in negotiation is pretty intolerant, aggressive, direct, hard-working and especially stubborn.

Nevertheless, in both Japan and Germany, people working in the international business usually speak English and communicate in this language.
}

2 Ces erreurs, portant sur des éléments dont la fréquence naturelle est assez élevée, ont été produites dans des conditions de surveillance maximale (productions écrites, souvent collectives, sans contrainte de temps, et avec possibilité d'utiliser dictionnaires et autres aides à la rédaction), par des étudiants (maîtrise LEA) ayant derrière eux 10 à 12 années d'apprentissage et, pour la plupart, au moins un séjour prolongé dans un pays anglophone. Malgré une exposition considérable aux données linguistiques brutes (données " positives»), et malgré les diverses corrections et explications qui ont dû leur être fournies tout au long de leur apprentissage (données "négatives»), ces erreurs subsistent, comme si ces apprenants restaient imperméables à l'évidence qui aurait pu les conduire à abandonner des hypothèses visiblement erronées. 
On peut toutefois se demander si les données en question leur sont réellement visibles; pour être utiles à l'apprenant, les données auxquelles il est exposé doivent être "perçues" (Gass \& Selinker 1994) et "traitées" (Ying 1995). Mais comment seront perçues et traitées des données dont le statut peut être ambigu ? En effet, si la distinction entre données « positives » et données « négatives » est claire pour le chercheur (voir par exemple le schéma proposé par Long, à paraître, cité par Oliver, 1995), elle ne l'est pas nécessairement pour l'apprenant, et ce pour deux raisons.

Du côté des données « positives » d'abord, qu'elles soient authentiques ou non, leur statut suppose qu'elles aient été "homologuées", soit de façon axiomatique parce que provenant d'un locuteur natif, soit de façon plus institutionnelle parce qu'étant fournies par une méthode ou par un professeur. Cela peut poser deux problèmes. Premièrement, il y a le risque de voir homologués par l'institution des emplois qui sont en réalité erronés ; ce cas semble heureusement assez rare, mais dans le domaine des connecteurs on pense au tristement célèbre « According to me... » (Lacoste \& Marcellin, $1984: 107)$, et il n'est pas impossible que l'utilisation abusive de «indeed» soit d'origine similaire. Deuxièmement, les données auxquelles est exposé un apprenant en milieu institutionnel proviennent souvent de sources douteuses : de ses propres productions, de celles de ses pairs et d'autres utilisateurs non natifs. Que fait-il de ces données non homologuées?

Du côté des données " négatives ", ensuite, on aura remarqué que toutes les erreurs citées plus haut sont des erreurs de contexte. Il n'y a pas de formes inacceptables, mais des associations incongrues. Les retours négatifs que l'apprenant est susceptible de recevoir seront donc sporadiques et liés au contexte d'emploi. Quelle généralisation fait-il à partir de ces corrections (et des cas, sans doute nombreux, de non-correction)?

C'est en partie pour répondre à ces questions qu'on s'intéresse de plus en plus aux démarches visant à améliorer la perception des données par l'apprenant : «consciousnessraising» (Rutherford 1987) ; "input enhancement» (Sharwood-Smith 1993); "input processing " (Van Patten \& Cadierno 1993) ou qu'on se sert des erreurs produites par l'apprenant pour l'amener à abandonner une généralisation erronée (Tomasello \& Herron 1989 ; Herron 1991). Pour une discussion de ces démarches, voir Ellis (1995). Dans cette optique, les outils de concordance peuvent apporter une aide précieuse. Ils rendent plus "saillantes" les caractéristiques que l'on veut faire percevoir; ils rapprochent les contextes d'occurrence les uns des autres et permettent en quelque sorte de constituer un « concentré » de données, dans lequel les régularités, souvent complexes et difficiles à résumer sous forme d'une « règle », seront plus perceptibles.

\section{Utilisation de l'outil de concordance}

7 Les concordances se prêtent à de nombreuses utilisations: voir notamment Sinclair (1991) et, pour des applications pédagogiques, Tribble \& Jones (1990). La démarche qui sera décrite ici est simple : pendant un semestre, les étudiants ont fourni, avec le texte papier de leurs devoirs (fondés sur des études de cas tirés de Ellis \& Williams, 1995), la disquette sur laquelle ils avaient préparé leur texte. À partir de ces fichiers textes, une concordance a été construite à l'aide du logiciel "Conc.» (Thomson 1992). Quelques erreurs récurrentes ont ensuite été sélectionnées pour faire l'objet d'un travail explicite, qui s'appuyait sur des séries d'exemples, mélangeant emplois acceptables et emplois erronés, tirées de la concordance (voir figure 1). Ces exemples étaient imprimés et 
distribués aux étudiants. Il leur était demandé de décider, individuellement, pour chaque occurrence du mot en caractères gras, si son utilisation était acceptable ou non. Ils ont ensuite comparé leurs jugements et, le cas échéant, ont proposé des corrections.

figure 1

152 brand image which is really important and necessary if it wants to reinforce

211 nuances. Nuances are important in the translation of the different

372 priorities (from 1, not important, to 10, extremely important). These

372 important, to 10 , extremely important). These also have to be considered

390 the United States, it had an important agent with who it merged in 1991,

396 is that it does not require an important capital investment : you work with

403 We already stated how important it was for a company to 'move'

405 but should not be the last. An important firm has to look further if it wants

436 but it represents a quite important capital and manpower investment

482 : - a brand name. - important advertising budgets. - a training in

494 few proper funds but with important views in international expansion, a

512 distribution but has to make important investments. It is not as risky as the

\section{Expérimentation}

8 L'expérimentation s'est faite avec deux groupes croisés. Pour certains des emplois problématiques (par exemple, l'utilisation de «the»), le premier groupe a suivi la démarche décrite plus haut, tandis que d'autres types d'erreurs (par exemple, dans l'utilisation de "important », «interesting» et "indeed»), la correction était faite par annotation des textes papier, qui étaient rendus aux étudiants en début de séance, avec un temps réservé pour la lecture des commentaires et pour d'éventuelles questions. Ces deux traitements des erreurs étaient inversés pour le groupe 2. Par la suite, les textes produits par les deux groupes avant la séance de correction ont été comparés avec ceux produits quelques semaines plus tard, afin de déterminer, toujours à l'aide de l'outil de concordance, s'il y avait eu évolution dans l'utilisation des éléments en question. Les résultats de cette comparaison sont résumés dans les tableaux 1 et 2 . 
Tableau 1. Groupe 1

\begin{tabular}{|l|l|l|l|}
\hline & occurrences & acceptables & inacceptables \\
\hline important & 15 & 15 & \\
\hline & 18 & 13 & 5 \\
\hline indeed & 6 & 1 & 5 \\
\hline & 8 & 2 & 6 \\
\hline interesting & 1 & & 1 \\
\hline & 0 & & \\
\hline the & 610 & 589 & 21 \\
\hline & 698 & 689 & 9 \\
\hline
\end{tabular}

$1^{\text {ère }}$ série de productions (sur fond ombré) : 10411 mots ; $2^{\mathrm{e}}$ série de productions (sur fond blanc) : 13943 mots

Tableau 2. Groupe 2

\begin{tabular}{|l|l|l|l|}
\hline & occurrences & acceptables & inacceptables \\
\hline important & 15 & 7 & 8 \\
\hline & 6 & 5 & 1 \\
\hline indeed & 6 & 0 & 6 \\
\hline & 1 & 1 & \\
\hline interesting & 5 & 1 & 4 \\
\hline & 3 & 2 & 1 \\
\hline the & 406 & 384 & 22 \\
\hline & 400 & 374 & 26 \\
\hline
\end{tabular}

\rceil$^{\text {ère }}$ série de productions (sur fond ombré) : 6721 mots ; 2 e série de productions (sur fond blanc) : 7636 mots

9 Ce qui ressort immédiatement de cette comparaison, c'est le peu d'effet produit par une correction traditionnelle. Les erreurs corrigées de cette façon (utilisation de "important ", etc. pour le groupe 1 ; de " the " pour le groupe 2) ont très peu évolué, et plutôt dans le mauvais sens. Corriger un texte n'est pas corriger une erreur. Quant aux erreurs traitées par concordance, l'évolution, si elle n'est pas spectaculaire, est néanmoins perceptible. 

conscience semble se traduire surtout par l'évitement: le nombre d'occurrences acceptables reste stable, mais le nombre total d'occurrences a baissé. Dans le cas de « indeed ", l'évitement est certainement la meilleure stratégie à adopter, car ce mot a une fréquence d'emploi anormalement élevée dans les productions d'étudiants francophones (Osborne 1994). Quant à « important » et «interesting ", on peut se demander si l'évitement a profité à d'autres adjectifs, mieux appropriés. Cela ne semble pas être le cas ici, si l'on excepte une très légère progression de « major » et de " promising », respectivement, dans des contextes comparables (« major investments », « promising markets »).

11 S'agissant de "the ", maintenant, on constate une baisse de fréquence dans les deux groupes (de presque 6 occurrences pour 100 mots à un peu plus de 5). Cette baisse est sans doute largement fortuite ; si l'on supprime toutes les références à « the UK » et « the USA », dont il est beaucoup question dans la première série de productions, on retrouve des fréquences beaucoup plus proches entre les deux séries. En revanche, les emplois abusifs de « the » ont assez fortement diminué dans le groupe 1 (qui a travaillé ce point à partir d'exemples tirés de la concordance), alors qu'ils ont légèrement progressé dans le groupe 2.

\section{Conclusion}

Le but de cette expérimentation était de voir si l'utilisation d'un outil de concordance pouvait aider à rendre les étudiants plus sensibles aux données de la langue, au point d'avoir un effet sur leurs productions. De ce point de vue, les résultats sont encourageants, même s'ils ne garantissent pas une amélioration durable ni une amélioration qui s'étendra à des styles moins surveillés. Il faut reconnaître aussi qu'une démarche expérimentée - ou « testée » plutôt - sur une si petite échelle n'apporte pas la preuve de son efficacité. Cette démonstration reste à faire, mais les résultats semblent déjà prometteurs.

13 Une prochaine étape consistera à mieux intégrer l'outil de concordance dans un processus de production-(correction)-révision assisté par ordinateur. Une fenêtre de concordance, après tout, n'est pas autre chose que le résumé d'un parcours hypertextuel. L'exploitation de liens hypertextuels dans des textes d'apprenants est assez récente (voir par exemple la démonstration proposée par Bowers ${ }^{1}$ ), mais elle offre d'intéressantes possibilités pour inciter l'étudiant à jeter un regard neuf sur ses productions et à profiter pleinement des renseignements qu'elles peuvent lui apporter. Les concordances, permettant aux apprenants de voir des rapprochements et des comparaisons à l'intérieur de leurs propres productions et entre celles-ci et des textes authentiques, apportent un outil de plus pour diversifier et enrichir les données à leur disposition. 


\section{BIBLIOGRAPHIE}

Ellis, N. 1995. « Consciousness in second language acquisition : A review of field studies and laboratory experiments ». Language Awareness 4, 123-146.

Ellis, J. \& D. Williams. 1995. International Business Strategy. Londres : Pitman.

Gass, S. \& L. Selinker. 1994. Second Language Acquisition: An introductory course. Hilldale : Lawrence Erlbaum.

Herron, C. 1991. "The Garden Path correction strategy in the foreign language classroom ». The French Review, 64, 966-977.

Lacoste, B. \& J. Marcelin. 1984. OK! $3^{\text {ème }}$. Paris : Nathan.

Long, M. (à paraître) Task-based Language Teaching. Oxford : Blackwell.

Oliver, R. 1995. « Negative feedback in child NS-NNS conversation ». Studies in Second Language Acquisition 17, 459-481.

Osborne, J. 1994. «La cohésion dans les productions écrites d'étudiants en anglais de spécialité : un problème culturel ?». ASp 5-6, 205-215.

Rutherford, W. 1987. Second Language Grammar: Learning and teaching. Londres : Longman. Selinker, L. 1974. « Interlanguage ». In Richards, Jack (ed.), Error Analysis. Harlow : Longman. Sharwood-Smith, M. 1993. «Input enhancement in instructed SLA: Theoretical bases ». Studies in Second Language Acquisition 15, 165-179.

Sinclair, J. 1991. Corpus, Concordance and Collocation. Oxford : Oxford University Press.

Thomson, J. 1992. Conc. A Concordance Generator. Dallas, TX : The Summer Institute of Linguists.

Tomasello, M. \& C. Herron. 1989. « Feedback for language transfer errors: The Garden Path technique ». Studies in Second Language Acquisition 11, 385-395.

Tribble, C. \& G. Jones. 1990. Concordances in the Classroom. Harlow : Longman.

VanPatten, B. \& T. Cadierno. 1993. «Input processing and SLA: A role for instruction ». The Modern Language Journal 77, 45-57.

Ying, H. 1995. « What sort of input is needed for intake? ». International Review of Applied Linguistics 33/3, 175-194.

\section{NOTES}

1. http://www.tnis.net/rbowers/demo.html 


\section{RÉSUMÉS}

Appliqués à la correction de textes d'apprenants, les outils de concordance servent à rendre plus saillantes certaines caractéristiques de leurs productions. L'utilisation de ces outils peut ainsi enrichir les données (négatives) fournies aux étudiants. D'après les premières indications de l'expérimentation décrite ici, cette démarche paraît produire plus d'effet sur les productions écrites que des techniques de correction traditionnelles.

The use of a concordancer as an aid to providing feedback on learners' written work may enhance (negative) input, by making certain patterns of language use more salient. First results of the procedure described here suggest that it is more effective than conventional correction techniques at reducing errors in written production.

\section{INDEX}

Mots-clés : concordance, enrichissement des données, erreur fossilisée, grammaire

Keywords : concordancer, fossilised error, language awareness, input enhancement

\section{AUTEUR}

\section{JOHN OSBORNE}

John Osborne est maître de conférences à l'Université de Savoie, où il enseigne la grammaire et la linguistique anglaises. Ses recherches portent sur la compétence métalinguistique dans l'apprentissage d'une langue seconde.john.osborne@univ-savoie.fr 